\title{
Adaptation of a flocculent Saccharomyces cerevisiae strain to lignocellulosic inhibitors by cell recycle batch fermentation
}

\author{
Roberto Landaeta $^{\mathrm{a}, *}$, Germán Aroca ${ }^{\mathrm{a}}$, Fernando Acevedo ${ }^{\mathrm{a}}$, José A. Teixeira ${ }^{\mathrm{b}}$, Solange I. Mussatto ${ }^{\mathrm{b}}$ \\ ${ }^{a}$ School of Biochemical Engineering, Pontificia Universidad Católica de Valparaíso, Gral. Cruz 34, Valparaíso, Chile \\ ${ }^{\mathrm{b}}$ Institute for Biotechnology and Bioengineering, Centre of Biological Engineering, University of Minho, Campus de Gualtar, $4710-057$ Braga, Portugal
}

\section{H I G H L I G H T S}

- A flocculent S. cerevisiae strain was adapted to lignocellulosic inhibitors.

- Five media simulating hydrolysates with six inhibitors were used for adaptation.

- Cell recycle batch fermentation was performed during 39 consecutive days.

- The adapted strain had increased growth and inhibitor transformation rates.

\section{A R T I C L E I N F O}

\section{Article history:}

Received 1 February 2012

Received in revised form 16 May 2012

Accepted 15 June 2012

Available online xxxx

\section{Keywords:}

Bioethanol

Saccharomyces cerevisiae

Inhibitors

Adaptation

Cell recycle batch fermentation

\begin{abstract}
A B S T R A C T
The ethanol production from lignocellulosic feedstocks is considered a promising strategy to increase global production of biofuels without impacting food supplies. However, some compounds released during the hydrolysis of lignocellulosic materials are toxic for the microbial metabolism, causing low ethanol yield and productivity during the fermentation. As an attempt to overcome this problem, the present study evaluated the adaptation of a flocculent strain of Saccharomyces cerevisiae (NRRL Y-265) to several inhibitory compounds usually found in lignocellulosic hydrolysates (acetic acid, furfural, hydroxymethylfurfural, vanillin, syringaldehyde, and hydroxybenzoic acid), aiming to minimize their negative effects on yeast metabolism, maximizing the ethanol production as a consequence. Cell recycle batch fermentation (CRBF) was performed during 39 consecutive days, using five different fermentation media with sequential increase in the concentration of inhibitory compounds, simulating the composition of lignocellulosic hydrolysates. This strategy allowed obtaining a yeast strain with increased ethanol volumetric productivity and growth rate (10\% and $70 \%$ respectively, over parent strain) able to produce ethanol with better results when cultivated in glucose-supplemented steam-exploded eucalyptus hydrolysate.
\end{abstract}

(c) 2012 Elsevier Ltd. All rights reserved.

\section{Introduction}

The ethanol production from lignocellulosic raw materials (2nd generation ethanol) is considered a promising strategy to increase the worldwide production of this biofuel without affecting the food provision. Therefore, great efforts have been directed in the last years to make feasible the ethanol production from these raw materials [1]. One of the main problems to be overcome in the industrial implementation of this production system is that during the pretreatment of the lignocellulosic materials to obtain sugar rich hydrolysates, several compounds that are toxic for the microbial metabolism are also released to the hydrolysates due to the use of high temperatures and chemicals (acids, bases, organic solvents, etc.) [2]. As a consequence, fermentation of sugar rich

\footnotetext{
* Corresponding author. Tel./fax: +56 978596020.

E-mail address: roberto.landaeta.1@mail.pucv.cl (R. Landaeta).
}

hydrolysates containing these inhibitory compounds is characterized by low ethanol yield and productivity [3]. Submitting the hydrolysate to a detoxification step prior to its use as fermentation medium has been proposed as an alternative to improve the fermentation results. However, this technology may require large investments, and the sugar losses may be significant according to the detoxification method used [2]. The yeast adaptation to the inhibitory compounds present in the hydrolysates could be an alternative to overcome this toxicity problem. Some studies have demonstrated that during the ethanol production from lignocellulosic feedstocks, the yeast Saccharomyces cerevisiae is able to convert several inhibitors to less-toxic derivatives at the cost of an extended lag phase and reduced ethanol productivity [4,5]. Cell adaptation has been suggested as being able to increase the inhibitor conversion rate, improving ethanol production [6]. Lignocellulose-derived inhibitors comprise several compounds that trigger a complex stress-related response in $S$. cerevisiae, involving 
interaction of several pathways and expression of genes with multiple functions [7]. Genetic engineering approaches for obtaining a inhibitor-tolerant yeast strain can have uncertain results in an industrial setting with wide-ranging inhibitor concentrations; for bioethanol production, directed-evolution techniques (which rely on selection pressure and spontaneous or induced mutations to obtain strains with augmented traits) could be more advantageous $[8,9]$.

An attractive variant for high cell density bioethanol production is self-immobilization or flocculation. Flocculating strains of S. cerevisiae have been used commercially in brewing since the 1980 s, as the flocculation step improves the separation of beer and reduces the cost of the process [10]. Flocculation expression is controlled by the FLO gene group, and depends on the FLO-mediated protein expression of membrane-type lectins, called flocculins, which bind to mannose residues present in the cell walls of cells $[11,12]$. Non-flocculating S. cerevisiae strains express the flocculent character under environmental stress conditions such as high ethanol levels, substrate depletion, and $\mathrm{pH}$ changes, and may develop strains that flocculate constitutively $[13,14]$. Up to date, two flocculation phenotypes (FloI and NewFlo) have been described in industrial strains, which differ in sensitivity to the culture conditions: the FloI phenotype is more stable to factors such as temperature, $\mathrm{pH}$, and presence of sugars in the culture medium, while the NewFlo phenotype is more sensitive to external conditions [15].

Flocculating yeasts are easily separated from the fermented broth, since the floc size is dependent of hydrodynamic conditions; in turn, floc size influences tolerance to chemical stresses and nutrient availability [16-18]. Thus, the manipulation of hydrodynamic conditions by the agitation speed in a stirred tank reactor can be used as a technique for replacing centrifugation of the broth, facilitating biomass retention in reactors, and modulating nutrient availability and resistance towards outside inhibitors for bioethanol production [19-21].

Based on the above-described considerations, the present study evaluated the adaptation of a flocculent strain of $S$. cerevisiae (NRRL Y-265) to inhibitory compounds present in lignocellulosic hydrolysates. Experimental assays were performed under conditions of cell recycle batch fermentation (CRBF), in which the flocculating ability of the yeast was used for the cells separation and use in the subsequent batch cultures. This fermentation technique is used in this work as an alternative to other directed-evolution approaches, such as sequential batches or growth in solid media [8]. The yeast was cultivated in five different fermentation media containing sequentially increased concentration of inhibitory compounds, aiming to obtain a strain suitable for use on ethanol production from lignocellulosic materials by conventional fermentation methods.

\section{Materials and methods}

\subsection{Microorganism and inoculum}

S. cerevisiae NRRL Y-265 (FloI phenotype) was the yeast strain used in the experiments. Cultures of this yeast were maintained at $4{ }^{\circ} \mathrm{C}$ in Petri dishes containing YM-agar medium with the following composition $(\mathrm{g} / \mathrm{L})$ : glucose (10), peptone (5), yeast extract (3) malt extract (3), and agar (20). To be used in the fermentation assays, one colony was recovered from the maintenance plate, cultivated in a new YM-agar medium at $30^{\circ} \mathrm{C}$ during $48 \mathrm{~h}$, and then one colony was inoculated in 250-mL Erlenmeyer flasks containing $50 \mathrm{~mL}$ of YM medium, which were maintained in an orbital shaker at $30{ }^{\circ} \mathrm{C}$ and $200 \mathrm{rpm}$, for $24 \mathrm{~h}$. After this time, the obtained inoculum was recovered and used in the fermentation assays. For liquid flask cultures, a constant inoculum volume was used (10\%).

\subsection{Fermentation media}

Fermentation media simulating the composition of lignocellulosic hydrolysates but with increasing concentration of inhibitor compounds were used for adaptation of the yeast strain. The base medium contained (g/L): glucose (25), yeast extract (1), $\mathrm{KH}_{2} \mathrm{PO}_{4}$ (1), $\left(\mathrm{NH}_{4}\right)_{2} \mathrm{SO}_{4}(0.4)$, and $\mathrm{MgSO}_{4} \times 7 \mathrm{H}_{2} \mathrm{O}(0.08)$, and was based in a medium reported for bioethanol production with flocculating yeast [22], but diluted 5-fold to lessen glucose repression and ethanol production during adaptation. This medium was supplemented with six inhibitory compounds commonly found in lignocellulosic hydrolysates: acetic acid, furfural, hydroxymethylfurfural (HMF), vanillin, syringaldehyde, and hydroxybenzoic acid. Five variations of this medium were made by increasing the concentration of all the six inhibitors in equal step changes (Table 1). The most concentrated medium (E) contained the inhibitory compounds in concentrations similar to those found in wood hemicellulosic hydrolysate [23].

\subsection{Cell recycle batch fermentation (CRBF) for the yeast adaptation}

Fermentation assays for the yeast adaptation was performed in a 1.6-L stirred tank bioreactor (Autoclavable Benchtop Fermenter Type R'ALF, Bioengineering AG, Wald, Switzerland) equipped with two Rushton turbines, $\mathrm{pH}$ and temperature control. A large - diameter $(5 \mathrm{~mm})$ sampling port was used for biomass and glucose samples. To be used in the experiments, the reactor was previously sterilized at $121^{\circ} \mathrm{C}$ for $30 \mathrm{~min}$. All the fermentation runs were maintained at $30^{\circ} \mathrm{C}, 200 \mathrm{rpm}$, with microaeration of $0.06 \mathrm{vvm}$. A working volume of $600 \mathrm{~mL}$ was used in each assay, and the $\mathrm{pH}$ of the media was controlled to 4.3 by addition of $\mathrm{NaOH}$ or $\mathrm{HCl}$.

The yeast adaptation was initiated by adding $60 \mathrm{~mL}$ of inoculum (Section 2.1) in $540 \mathrm{~mL}$ of culture medium A, for an initial biomass concentration of $0.1 \mathrm{~g} / \mathrm{L}$. The batch was maintained until more than $90 \%$ of the initial glucose was consumed. Then, the agitation was stopped for $15 \mathrm{~min}$ to allow the biomass flocculation and the fermented broth separation. The fermented broth $(540 \mathrm{~mL})$ was removed from the vessel by pumping it out through the outlet pipe, taking care to not disturb the biomass at the bottom of the reactor. A volume of $60 \mathrm{~mL}$ of the fermented broth was maintained inside the reactor to be used as inoculum for the subsequent batch. Then, $540 \mathrm{~mL}$ of sterile medium A was loaded again to the reactor and the agitation was resumed when the loading ended, marking the start of a new batch. Duplicate samples were taken every 8$12 \mathrm{~h}$ for biomass and glucose measurement, accounting to 2-3 samples per batch. Each batch lasted $24 \mathrm{~h}$ approximately, and media replacement was made every day within a time interval of $2-3 \mathrm{~h}$, reducing the possibility of starvation periods.

At least four successive batches were made to each fermentation medium prior to change to the following medium with increased inhibitor levels. Adaptation was monitored by calculating the specific maximum glucose consumption rate of each batch, measured at $10 \%$ or less of the initial glucose remaining in the reactor. After the CRBF adaptation, the obtained strain (Y-265A3) was cultivated on YM agar plates supplemented with $1 \mathrm{~g} / \mathrm{L}$ furfural and $4 \mathrm{~g} / \mathrm{L}$ acetic acid for maintenance and further evaluation, since culture in synthetic inhibitor-containing solid medium (E medium) proved unfeasible.

\subsection{Adapted strain evaluation}

The performance of the adapted and parent strains was compared by cultivation in the fermentation media $\mathrm{C}, \mathrm{D}$ and $\mathrm{E}$, and also by cultivation in a hydrolysate medium obtained from the liquid fraction of steam-exploded eucalyptus chips at $196^{\circ} \mathrm{C}$ for $9.5 \mathrm{~min}$. Since glucose content in the hydrolysate-derived liquor 
Table 1

Concentration of inhibitory compounds in the fermentation media used for adaptation of the yeast Saccharomyces cerevisiae.

\begin{tabular}{|c|c|c|c|c|c|}
\hline \multirow[t]{2}{*}{ Inhibitor compound } & \multicolumn{5}{|c|}{ Concentration in the fermentation medium $(\mathrm{g} / \mathrm{L})$} \\
\hline & A & B & $\mathrm{C}$ & $\mathrm{D}$ & $\mathrm{E}$ \\
\hline Acetic acid & 0.9 & 1.8 & 2.7 & 3.6 & 4.5 \\
\hline Furfural & 0.2 & 0.4 & 0.6 & 0.8 & 1 \\
\hline Hydroxymethylfurfural (HMF) & 0.04 & 0.08 & 0.12 & 0.16 & 0.2 \\
\hline Vanillin & 0.08 & 0.16 & 0.24 & 0.32 & 0.4 \\
\hline Syringaldehyde & 0.02 & 0.04 & 0.06 & 0.08 & 0.1 \\
\hline Hydroxybenzoic acid & 0.02 & 0.04 & 0.06 & 0.08 & 0.1 \\
\hline
\end{tabular}

was very low $(0.24 \mathrm{~g} / \mathrm{L})$ for ethanol production, it was supplemented with this carbon source to attain $110 \mathrm{~g} / \mathrm{L}$ of glucose, and was also enriched with the nutrients $(\mathrm{g} / \mathrm{L})$ : yeast extract (1), $\mathrm{KH}_{2} \mathrm{PO}_{4}(5),\left(\mathrm{NH}_{4}\right)_{2} \mathrm{SO}_{4}(2)$, and $\mathrm{MgSO}_{4} \times 7 \mathrm{H}_{2} \mathrm{O}(0.4)$, to obtain an ethanol production medium [22].

Fermentation runs were carried out in $250-\mathrm{mL}$ Erlenmeyer flasks containing $50 \mathrm{~mL}$ medium (media C, D, and E), and in 500$\mathrm{mL}$ Erlenmeyer flasks containing $200 \mathrm{~mL}$ medium (hydrolysate medium), in an orbital shaker at $200 \mathrm{rpm}, 30^{\circ} \mathrm{C}$ for $48 \mathrm{~h}$ or $100 \mathrm{~h}$, respectively. The adapted strain was inoculated using the same procedure indicated in Section 2.1.

\subsection{Analytical techniques}

For CRBF adaptation and strain verification in synthetic media, biomass, glucose and ethanol concentrations were estimated spectrophotometrically using a 96-well microplate reader (Biotech Synergy HT). Biomass samples ( $20 \mathrm{~mL}$ in bioreactor, $1 \mathrm{~mL}$ flasks) were taken with a micropipette equipped with a large-opened cut tip, and then deflocculated by stirring vigorously with the addition of EDTA $75 \mathrm{mM}$ at 1:1 volume. Then, the cell suspension was diluted with tap water, and the absorbance at $620 \mathrm{~nm}$ was determined. The biomass concentration was calculated through a dry mass calibration curve obtained from cells cultivated in liquid culture as previously described (Section 2.1). Glucose concentration was determined by the DNS method [24], and the ethanol concentration by using an enzymatic kit (Boehringer Ethanol Test Kit, R-Biopharm). Both methods were adapted for use in 96-well microplates (final volume $0.3 \mathrm{~mL}$ ). All samples were analyzed in duplicates.

Samples taken from the strain verification in hydrolysate liquor were analyzed by HPLC using a BioRad Aminex HPX-87H column. Glucose, ethanol, and acetic acid concentrations were measured with a refractive index detector, at $45{ }^{\circ} \mathrm{C}$, and $5 \mathrm{mM} \mathrm{H}_{2} \mathrm{SO}_{4}$ as eluent in a flow rate of $0.6 \mathrm{~mL} / \mathrm{min}$. Furfural, HMF and syringaldehyde were measured with a UV detector at $210 \mathrm{~nm}, 65^{\circ} \mathrm{C}$, and a mixture of $90: 105 \mathrm{mM} \mathrm{H}_{2} \mathrm{SO}_{4}$ :acetonitrile as eluent in a flow rate of $0.6 \mathrm{~mL} /$ min.

\section{Results and discussion}

\subsection{Yeast adaptation}

The yeast cultivation by CRBF was carried for 39 days, during which 38 batches were completed (Fig. 1). Each batch consumed more than $90 \%$ glucose, and almost all of them did have less than $1 \mathrm{~g} / \mathrm{L}$ remaining at the end. The biomass content in the reactor increased steadily in the first eight batches (media A and B), reaching approx. $3 \mathrm{~g} / \mathrm{L}$. Batches in medium $\mathrm{C}$ presented unstable biomass measurements, and biomass growth stopped almost entirely in batches carried out in media $\mathrm{D}$ and $\mathrm{E}$, causing a decrease in biomass concentration as each batch removed non-flocculated biomass. In medium E, each batch started and ended with approx. $2 \mathrm{~g} / \mathrm{L}$ of biomass, resulting in a near-zero growth rate (acknowledging data spread). However, glucose continued to be consumed (Fig. 2). After seven batches in medium $\mathrm{E}$, the culture was reverted to medium $\mathrm{C}$ to allow biomass growth under less stressful conditions. The adaptation protocol was resumed, changing to media D and $\mathrm{E}$ after four successive batches in each medium. Biomass concentration increased slightly in the last batches made in medium D, but descended in subsequent batches in medium E. Glucose exhibited high variability, probably due to dilution by broth exchange, detection of additional reducing carbohydrates in the medium and possible interference by EDTA on DNS method.

Lignocellulosic inhibitory compounds exert different effects over cellular metabolism, due to present different mechanisms of inhibition. Furfural, for example, which is one of the main inhibitory compounds in lignocellulosic hydrolysates, has been reported to reduce cell growth in S. cerevisiae, but metabolic activity is less affected [5]. This could explain the reduction observed in cell growth while the glucose consumption was maintained.

Average specific glucose consumption rates increased during the CRBF adaptation (Fig. 2), changing from $0.51 \pm 0.14 \mathrm{~g} / \mathrm{g} \mathrm{h}$ in the first batches on medium $B$, to $0.79 \pm 0.11 \mathrm{~g} / \mathrm{g}$ h in the last stage of adaptation on medium E. Although ethanol was not measured during the CRBF adaptation, an increase in glucose consumption rate could be related to a more tolerant strain, since glucose consumption rate is negatively affected by inhibitors [25].

\subsection{Evaluation of the adapted strain}

To evaluate the ability of the new strain (designated as Y265A3) for ethanol production, batch cultures of the adapted and original strains were firstly performed in the formulated media $\mathrm{C}$

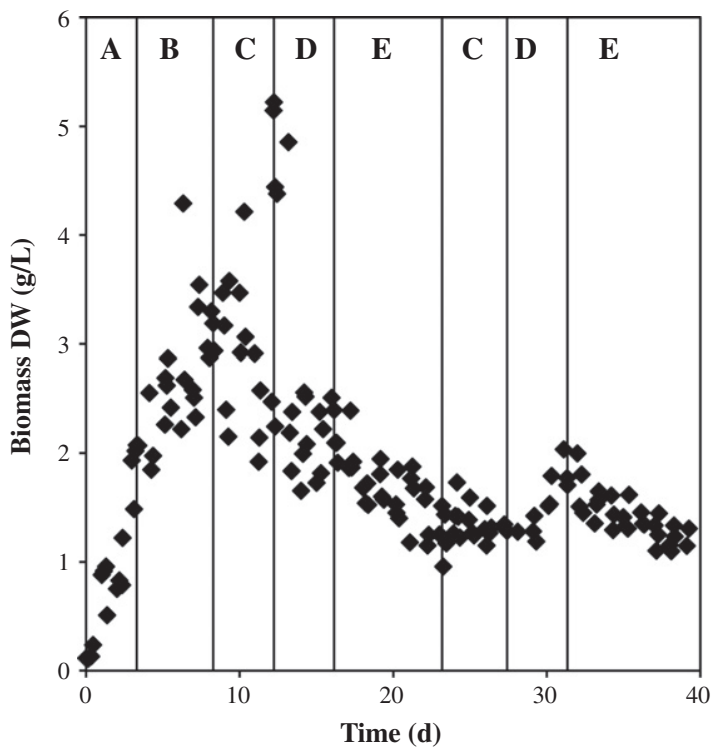

Fig. 1. Biomass growth during adaptation of S. cerevisiae NRRL Y-265 to lignocellulosic inhibitors by cell recycle batch fermentation (CRBF). Vertical solid lines mark changes in the fermentation medium (A-E). 


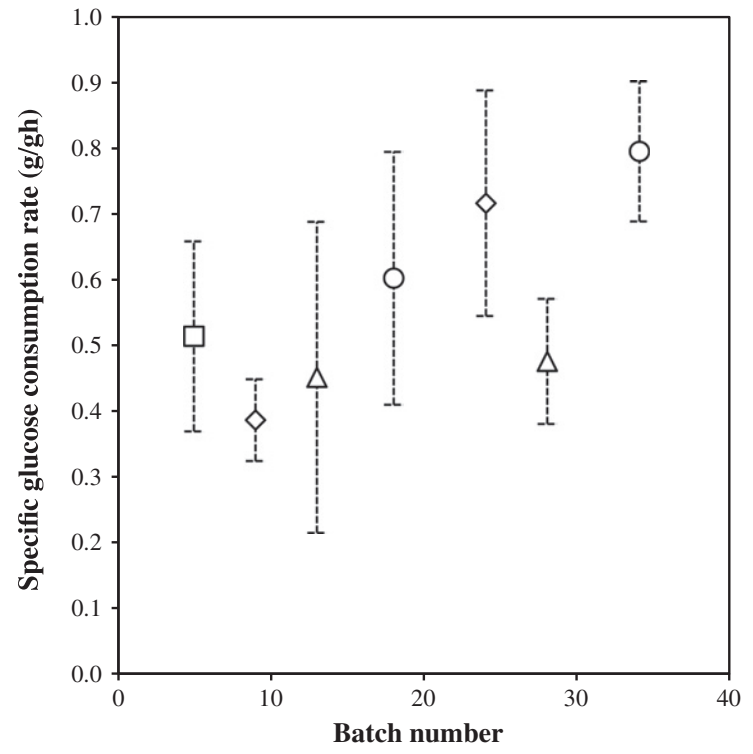

Fig. 2. Specific glucose consumption rate (average values) during the adaptation of S. cerevisiae NRRL Y-265 by cell recycle batch fermentation. Error bars represent average standard deviation of specific glucose consumption rates for all the batches in each medium. Fermentation medium: B, square; C, lozenge; D, triangle; E, circle.

and D. Fig. 3 shows the glucose consumption and biomass growth for both strains. At moderate inhibitor levels (medium $\mathrm{C}$ ), both control and adapted strains had similar behaviors, achieving ethanol yields of 0.3 and $0.29 \mathrm{~g} / \mathrm{g}$ glucose respectively (60\% and $56 \%$ of the maximum theoretical value), and similar volumetric productivities and growth rates (Table 2). At moderately high inhibitor levels (medium D), however, a marked increase on ethanol production was verified for the adapted strain (Y-265A3), with an ethanol volumetric productivity of $0.13 \mathrm{~g} / \mathrm{L} \mathrm{h}$ after $49 \mathrm{~h}$, which was $50 \%$ higher than that achieved by the non-adapted strain. Ethanol yield was also higher for the adapted strain. On the other hand, the biomass growth rate was very low for both strains, probably due to the high inhibitor level. When the strains were cultivated in medium E, there was a small amount of glucose consumption, but no significant growth even after $48 \mathrm{~h}$ of cultivation (data not shown), indicating that for a critical inhibitor level, growth rate is reduced to near zero. Glycerol, $\mathrm{CO}_{2}$ and other metabolic byproducts were not measured in these experiments, so carbon balances could not be made with enough certainty. In the case of batch culture made in medium $C(0.3 \mathrm{~g} / \mathrm{g}$ ethanol yield $)$ it is possible that the higher glycerol production could have explained the low theoretical ethanol yield.

To further evaluate the performance of the adapted strain for ethanol production, the above experience was repeated but changing the fermentation media $C$ and $D$ to a steam-exploded eucalyptus hydrolysate, supplemented with glucose and nutrient sources (Fig. 4). When compared to the previous experiments, it was observed an extension of the lag phase (almost twice bigger) for both strains in hydrolysate medium, this phase being slightly smaller for the Y-265A3 strain. Growth rate and ethanol productivity were higher for the adapted strain ( $70 \%$ and $10 \%$, respectively) but glucose was not completely metabolized and there was no difference in ethanol yield (Table 3 ). Since the concentration of inhibitory compounds in the hydrolysate medium was similar to that used in the formulation of medium C (Fig. 5), except that it contained lower levels of vanillin $(<0.05 \mathrm{~g} / \mathrm{L}$ ) and hydroxybenzoic acid (trace), the increased lag phase, residual glucose and lower growth rate observed when the yeasts were cultivated in this medium suggest the presence of other inhibitor compounds in the hydrolysate, which were
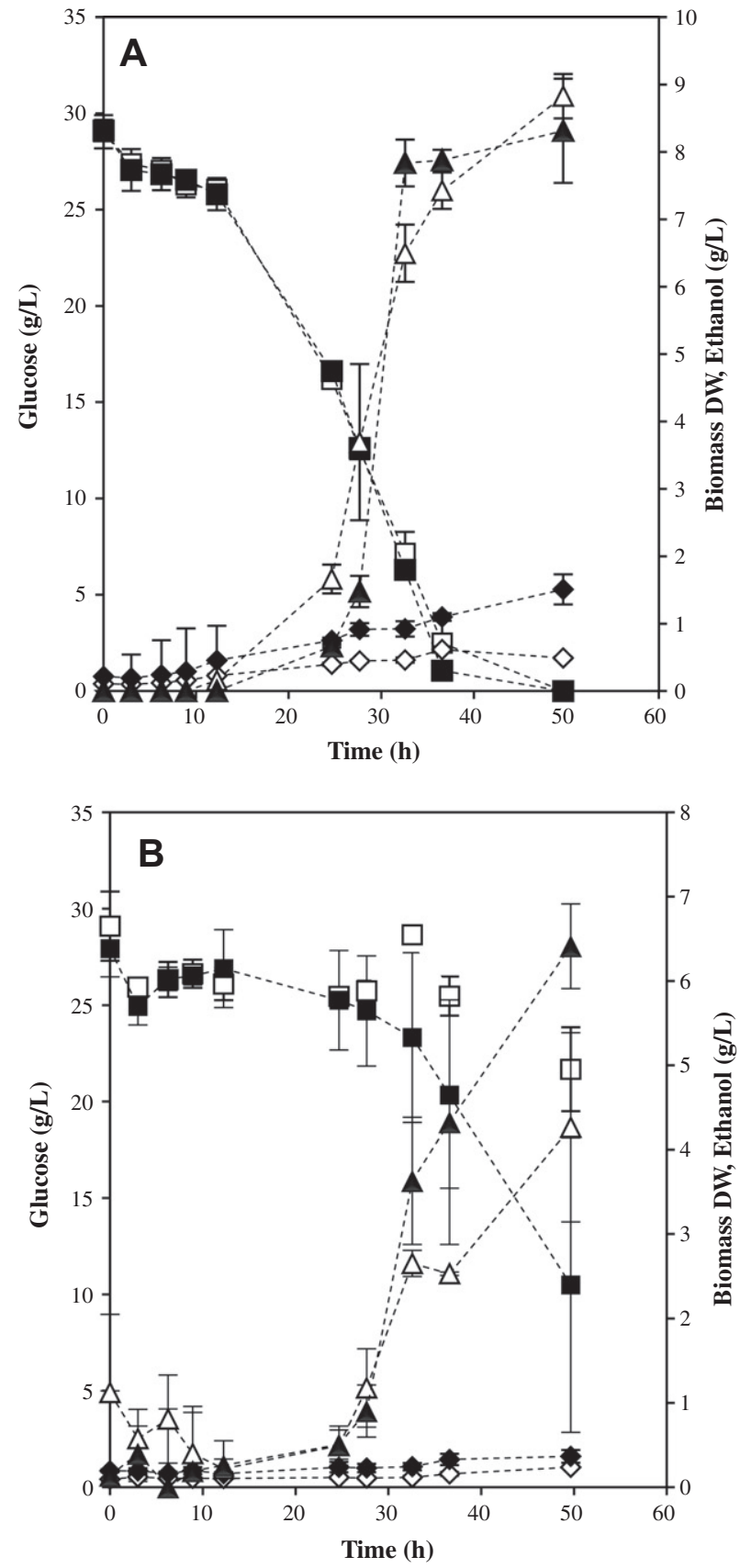

Fig. 3. Batch cultures of non-adapted S. cerevisiae NRRL Y-265 (white), and adapted S. cerevisiae Y-265A3 (black). (A) Medium C and (B) medium D. Glucose, square; biomass, lozenge; ethanol, triangle. Error bars represent sample spread.

Table 2

Culture parameters obtained by cultivation of the yeasts S. cerevisiae NRRL Y-265 (non-adapted) and Y-265A3 (adapted) in fermentation media C and D.

\begin{tabular}{llllll}
\hline Strain & Y-265 & & & Y-265A3 & \\
\cline { 2 - 3 } Medium & $\mathrm{C}$ & $\mathrm{D}$ & & $\mathrm{C}$ & $\mathrm{D}$ \\
\hline Specific growth rate $(1 / \mathrm{h})$ & 0.06 & 0.03 & & 0.07 & 0.02 \\
Ethanol productivity $(\mathrm{g} / \mathrm{L} \mathrm{h})$ & 0.18 & 0.09 & & 0.17 & 0.13 \\
Ethanol yield $(\mathrm{g} / \mathrm{g})$ & 0.3 & 0.15 & & 0.29 & 0.24 \\
Biomass yield $(\mathrm{g} / \mathrm{g})$ & 0.01 & 0.02 & & 0.03 & 0.02 \\
\hline
\end{tabular}

not evaluated in the present study. In fact, although acetic acid, furfural, HMF, vanillin, syringaldehyde, and hydroxybenzoic acid are the inhibitory compounds most commonly found in lignocellulosic 


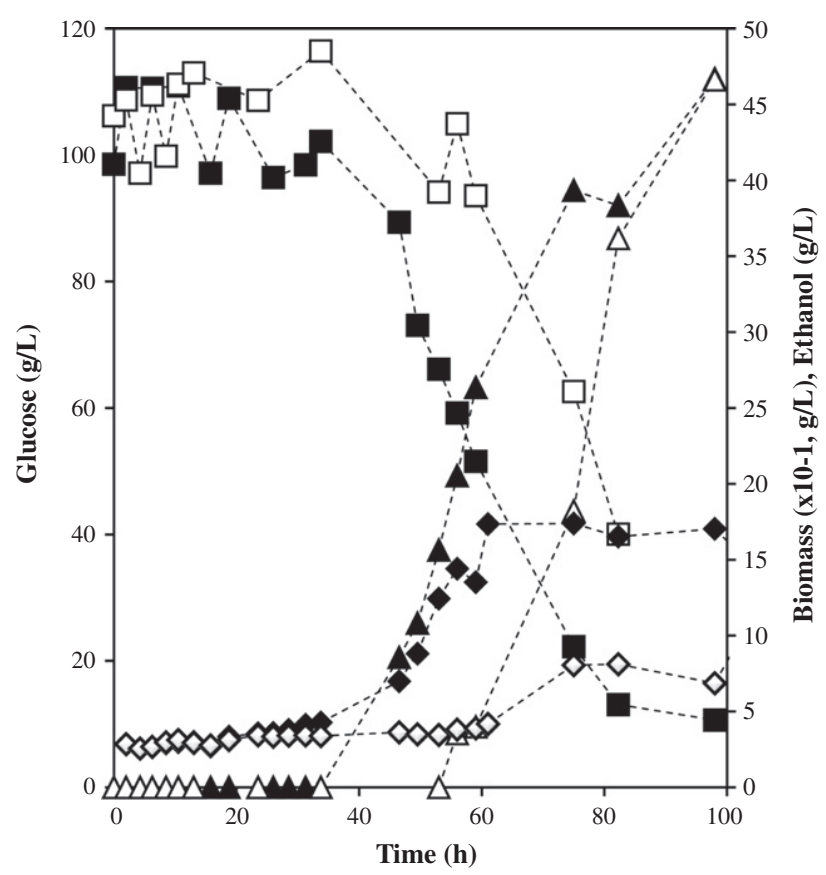

Fig. 4. Batch cultures of non-adapted S. cerevisiae NRRL Y-265 (white), and adapted Y-265A3 (black) in steam-exploded eucalyptus hydrolysate. Glucose, square; biomass, lozenge; ethanol, triangle.

Table 3

Culture parameters obtained by cultivation of the yeasts S. cerevisiae NRRL Y-265 (non-adapted) and Y-265A3 (adapted) in fermentation media based on steamexploded eucalyptus hydrolysate. Volumetric ethanol productivities were calculated dividing the ethanol concentration in each sample by its corresponding batch time. The maximum value is showed.

\begin{tabular}{lll}
\hline Yeast strain & Y-265 & Y265A3 \\
\hline Specific growth rate $(1 / \mathrm{h})$ & 0.03 & 0.05 \\
Maximum ethanol volumetric productivity $(\mathrm{g} /$ & 0.48 (at & 0.53 (at \\
$\mathrm{L} \mathrm{h})$ & $98 \mathrm{~h})$ & $75 \mathrm{~h})$ \\
Ethanol yield $(\mathrm{g} / \mathrm{g})$ & 0.48 & 0.48 \\
\hline
\end{tabular}

hydrolysates, a variety of other inhibitors may also be present in this medium according to the acid concentration, temperature and other conditions used for hydrolysis, and such compounds may also affect the metabolism of the microorganism used for fermentation [2], specially when batch fermentations did not have $\mathrm{pH}$ control or buffer. Interestingly, the ethanol yield obtained in the hydrolysate was higher than the attained in $\mathrm{C}$ medium. As a general rule, higher inhibitor concentration means increased lag time and decreased biomass ethanol concentration, leading to lower ethanol productivity. However, it has been cited that presence of acetic acid (c. $3 \mathrm{~g} / \mathrm{L}$ ) actually increases ethanol yield due to decreased glycerol and biomass yield [26].

It is important to emphasize that the adapted strain also showed an increased transformation rate for furfural, HMF and syringaldehyde, reaching basal levels of these inhibitors between 20 and $40 \mathrm{~h}$ earlier than the parent strain (Fig. 5). Acetic acid was not transformed over the course of the batch cultures. Similar profiles for these inhibitors and increased transformation rates for adapted strains have been reported $[27,28]$. Generally speaking, an adapted strain could have a higher growth rate in the presence of inhibitors. However, since furfural and HMF exert action over the lag phase of batch growth, an adapted strain might have attained, through directed evolution, a speedier biotransformation of inhibitors during lag phase (Fig. 5A), maintaining similar growth rate than the parent strain. An improved biotransformation rate is
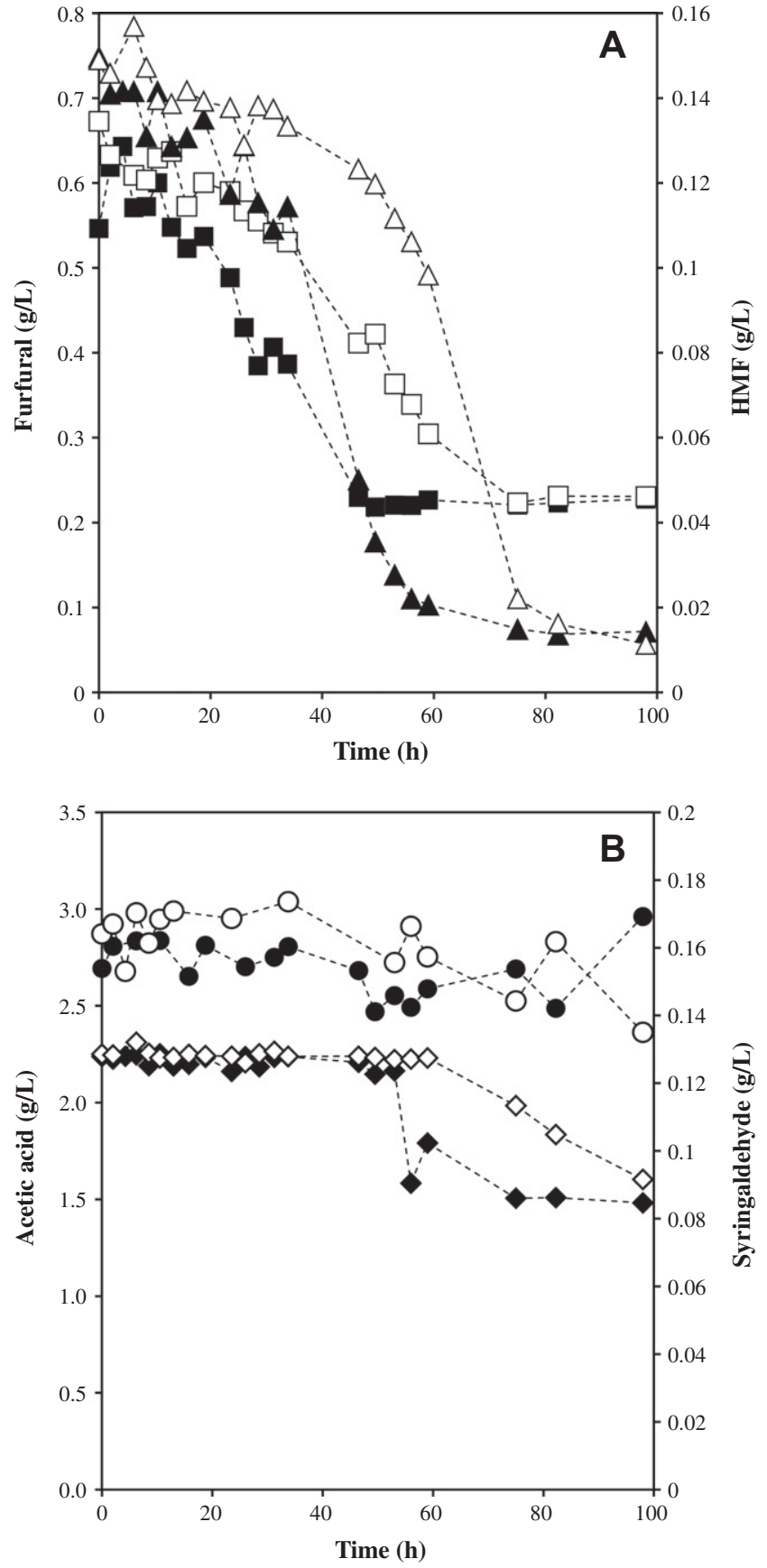

Fig. 5. Furfural, hydroxymethylfurfural (HMF), acetic acid, and syringaldehyde profiles from batch cultures of non-adapted S. cerevisiae NRRL Y-265 (white), and adapted Y-265A3 (black) in steam-exploded eucalyptus hydrolysate. Furfural, square; HMF, triangle; acetic acid, circle; syringaldehyde, lozenge.

crucial for in situ detoxification of lignocellulosic hydrolysates, replacing expensive detoxification steps in bioethanol production. Thus, the adapted strain can be considered suitable for bioethanol production.

Flocculating yeasts have been shown to be more resilient to environmental stresses (including lignocellulosic inhibitors) than non-flocculating yeast strains [19]. Their low growth rates could be related to the flocculating character, since the expression of flocculence in yeast cells results in marked differences in the overall genetic profile between flocculent and non-flocculent strains [29]. Low growth rates can also be found in flocculent yeast cultures due to mass-transfer limitations arising from glucose 
diffusivity inside the floc [30]; floc size can also affect growth rate and ethanol tolerance [18]. A diminished growth rate could hinder a successful adaptation to inhibitors but under the stirring conditions used in the experiment, biomass present as yeast flocs is subject to a dynamic process, involving floc formation and breakage during batch growth; flocculin-expressing (flocculating) cells are present in suspended single-cell form due to hydrodynamic stresses, low surface hydrophobicity and floc breakage by $\mathrm{CO}_{2}$ buildup [31]. Thus, during batch growth, suspended flocculin-expressing yeast can divide and adapt to inhibitors present in the medium. As non-flocculating cells are removed at the end of each batch, only the flocculating yeast population that was able to grow in the inhibitor-containing medium is retained in the bioreactor. During enough batch cycles, a sufficient amount of genetic changes arising from spontaneous mutations should buildup in the flocculating population. Nevertheless, the growth rate is of secondary importance for the ethanol yield and productivity. In the present study, although the ethanol yield for the adapted strain was not optimal ( $46 \%$ and $91 \%$ of the maximum theoretical value in medium $C$ and hydrolysate, respectively), the successful adaptation of the yeast in formulated culture media open up new possibilities for future research works.

Evolutionary engineering techniques such as used in this work have been used to create new strains adapted to lignocellulosic inhibitors. For example, Martín et al. [32] used a continuous culture with increased concentrations of sugarcane bagasse hydrolysate to obtain a $S$. cerevisiae strain with improved specific ethanol productivity ( 2.55 vs. $1.15 \mathrm{~g} / \mathrm{g}$ h at $50 \%$ hydrolysate); however, this strain also presented low biomass yield and decreased ethanol productivity on more inhibitory media. Keating et al. [33] generated a strain of $S$. cerevisiae $\mathrm{T} 1$ by adaptation through CRBF in media supplemented with furfural, HMF and acetic acid, and observed that the tolerance and culture parameters were also dose-dependent. Liu et al. $[6,34]$ engineered several $S$. cerevisiae clones for furfural and HMF tolerance, and obtained an adapted strain with better capacity for in situ detoxification of these inhibitors. In the present study, the obtained strain (Y265-A3) showed a reduced lag phase and a higher biotransformation rate compared to the parent strain in inhibitor-containing media. As batch lag time directly affects volumetric ethanol productivity, the adapted strain should be better for bioethanol production. Also, it is necessary to point out that this study presents results from only one CRBF culture. Strains obtained by adaptive evolution, while can converge to similar phenotypes, have different gene expression patterns due to random mutations during the adaptation [35], thus diminishing the usability of repeats for these cultures. To the best of our knowledge, the present study is the first report about evolutionary-engineered flocculating strains adapted to lignocellulosic inhibitors by means of a CRBF approach.

\section{Conclusions}

An adapted strain of the flocculating yeast $S$. cerevisiae NRRL Y-265 was obtained through adaptation by cell recycle batch fermentation. The exposure to increased levels of lignocellulosic inhibitors in fermentation media simulating lignocellulosic hydrolysates generated a tolerant strain after 39 days of successive batch culture, with ethanol productivity and yield increased by $50-60 \%$ in the presence of moderately high levels of inhibitors. When used in batch fermentation in the presence of inhibitors derived from steam-exploded eucalyptus, the adapted Y-265A3 strain showed higher ethanol productivity and growth rate, and higher transformation rates for furfural, HMF and syringaldehyde, when compared to the non-adapted strain, which can be considered an important advantage for bioethanol production.

\section{Acknowledgements}

This work was funded by CONICYT-MINERGIA, Chile (Programa Pasantías en Extranjero, Convocatoria 2010) and by Innova Chile Project 208-7320 Technological Consortium Bioenercel S.A.

\section{References}

[1] Mussatto SI, Dragone G, Guimarães PMR, Silva JPA, Carneiro LM, Roberto IC, et al. Technological trends, global market, and challenges of bio-ethanol production. Biotechnol Adv 2010;28:817-30.

[2] Mussatto SI, Roberto IC. Alternatives for detoxification of diluted-acid lignocellulosic hydrolyzates for use in fermentative processes - a review. Bioresource Technol 2004;93:1-10.

[3] Klinke HB, Olsson L, Thomsen AB, Ahring BK. Potential inhibitors from wet oxidation of wheat straw and their effect on ethanol production of Saccharomyces cerevisiae: wet oxidation and fermentation by yeast. Biotechnol Bioeng 2003;81:738-47.

[4] Larsson S, Quintana-Sáinz A, Reimann A, Nilvebrant NO, Jönsson LJ. Influence of lignocellulose-derived aromatic compounds on oxygen-limited growth and ethanolic fermentation by Saccharomyces cerevisiae. Appl Biochem Biotechnol 2000;84-86:617-32.

[5] Palmqvist E, Almeida JS, Hahn-Hägerdal B. Influence of furfural on anaerobic glycolytic kinetics of Saccharomyces cerevisiae in batch culture. Biotechnol Bioeng 1999;62:447-54.

[6] Liu ZL, Moon J, Andersh BJ, Slininger PJ, Weber S. Multiple gene-mediated $\mathrm{NAD}(\mathrm{P}) \mathrm{H}$-dependent aldehyde reduction is a mechanism of in situ detoxification of furfural and 5-hydroxymethylfurfural by Saccharomyces cerevisiae. Appl Microbiol Biotechnol 2008;81:743-53.

[7] Liu ZL. Molecular mechanisms of yeast tolerance and in situ detoxification of lignocellulose hydrolysates. Appl Microbiol Biotechnol 2011;90:809-25.

[8] Sauer U. Evolutionary engineering of industrially important microbial phenotypes. Adv Biochem Eng 2001;73:129-69.

[9] Çakar ZP, Seker UOS, Tamerler C, Sonderegger M, Sauer U. Evolutionary engineering of multiple-stress resistant Saccharomyces cerevisiae. FEMS Yeast Res 2005:5:569-78.

[10] Gong C, Cao N, Du J, Tsao G. Ethanol production from renewable resources. Adv Biochem Eng Biotechnol 1999;65:207-42.

[11] Verstrepen KJ, Derdelinckx G, Verachtert H, Delvaux FR. Yeast flocculation: what brewers should know. Appl Microbiol Biotechnol 2003;61:197-205.

[12] Verstrepen KJ, Klis FM. Flocculation, adhesion and biofilm formation in yeasts. Mol Microbiol 2006;60:5-15.

[13] Claro FB, Rijsbrack K, Soares EV. Flocculation onset in Saccharomyces cerevisiae: effect of ethanol, heat and osmotic stress. J Appl Microbiol 2007;102:693-700.

[14] Sampermans S, Mortier J, Soares EV. Flocculation onset in Saccharomyces cerevisiae: the role of nutrients. J Appl Microbiol 2005;98:525-31.

[15] Soares EV, Teixeira JA, Mota M. Effect of cultural and nutritional conditions on the control of flocculation expression in Saccharomyces cerevisiae. Can J Microbiol 1994;40:851-7.

[16] Purwadi R, Taherzadeh MJ, Brandberg T. A possible industrial solution to ferment lignocellulosic hydrolyzate to ethanol: continuous cultivation with flocculating yeast. Int J Mol Sci 2007;8:920-32.

[17] Vicente A, Teixeira JA, Dluhý M. Increase of ethanol productivity in an airlift reactor with a modified draught tube. Can J Chem Eng 1999;77:497-502.

[18] Xue C, Zhao XQ Bai FW. Effect of the size of yeast flocs and zinc supplementation on continuous ethanol fermentation performance and metabolic flux distribution under very high concentration conditions. Biotechnol Bioeng 2010;105:935-44.

[19] Lei J, Zhao X, Ge X, Bai FW. Ethanol tolerance and the variation of plasma membrane composition of yeast floc populations with different size distribution. J Biotechnol 2007;131:270-5.

[20] Xu T, Zhao X, Bai FW. Continuous ethanol production using self-flocculating yeast in a cascade of fermentors. Enzyme Microb Technol 2005;37:634-40

[21] Li F, Zhao X, Ge X, Bai FW. An innovative consecutive batch fermentation process for very high gravity ethanol fermentation with self-flocculating yeast. Appl Microbiol Biotechnol 2009;84:1079-86.

[22] Teixeira JA, Sousa ML, Mota M. Comparative analysis of ethanolic fermentation in two continuous flocculation bioreactors and effect of flocculation additive. Bioproc Eng 1994;11:83-90.

[23] Carvalho GB, Mussatto SI, Cândido EJ, Almeida JB. Comparison of different procedures for the detoxification of eucalyptus hemicellulosic hydrolysate for use in fermentative processes. J Chem Technol Biotechnol 2006;81:152-7.

[24] Miller GL. Use of dinitrosalicylic acid reagent for determination of reducing sugar. Anal Chem 1959;31:426-8.

[25] Lin FM, Tan Y, Yuan YJ. Temporal quantitative proteomics of Saccharomyces cerevisiae in response to a nonlethal concentration of furfural. Proteomics 2009;9:5471-83.

[26] Taherzadeh MJ. Acetic acid: friend or foe in anaerobic batch conversion of glucose to ethanol by Saccharomyces cerevisiae? Chem Eng Sci 1997;52:2653-9.

[27] Delgenes JP, Moletta R, Navarro JM. Effects of lignocellulose degradation products on ethanol fermentations of glucose and xylose by Saccharomyces cerevisiae, Zymomonas mobilis, Pichia stipitis, and Candida shehatae. Enzyme Microb Technol 1996;19:220-5. 
[28] Liu ZL, Slininger PJ, Gorsich SW. Enhanced biotransformation of furfural and hydroxymethylfurfural by newly developed ethanologenic yeast strains. Appl Biochem Biotechnol 2005;121-124:451-60.

[29] Smukalla S, Caldara M, Pochet N, Beauvais A, Guadagnini S, Yan C, et al. FLO1 is a variable green beard gene that drives biofilm-like cooperation in budding yeast. Cell 2008;135:726-37.

[30] Vicente A, Dluhý M, Ferreira EC, Mota M, Teixeira JA. Mass transfer properties of glucose and $\mathrm{O}_{2}$ in Saccharomyces cerevisiae flocs. Biochem Eng J $1998 ; 2: 35-43$.

[31] Stratford M. Yeast flocculation: a new perspective. In: Rose AH, editor. Advances in microbial physiology, vol. 33. London: Academic Press; 1992. p. $1-71$

[32] Martín C, Marcet M, Almazán O, Jönsson LJ. Adaptation of a recombinant xylose-utilizing Saccharomyces cerevisiae strain to a sugarcane bagasse hydrolysate with high content of fermentation inhibitors. Bioresource Technol 2007;98:1767-73.

[33] Keating JD, Panganiban C, Mansfield SD. Tolerance and adaptation of ethanologenic yeasts to lignocellulosic inhibitory compounds. Biotechnol Bioeng 2006;93:1196-206.

[34] Liu ZL, Ma M, Song M. Evolutionarily engineered ethanologenic yeast detoxifies lignocellulosic biomass conversion inhibitors by reprogrammed pathways. Mol Genet Genomics 2009;282:233-44.

[35] Fong SS, Joyce AR, Palsson B. Parallel adaptive evolution cultures of Escherichia coli lead to convergent growth phenotypes with different gene expression states. Genome Res 2005;15:1365-72. 